Diabetologia (1994) 37: 897-904

\title{
The association of the insulin resistance syndrome with impaired glucose tolerance and NIDDM in the Japanese general population: the Hisayama study
}

\author{
T. Ohmura, K. Ueda, Y. Kiyohara, I. Kato, H. Iwamoto, K. Nakayama, K. Nomiyama, S. Ohmori, T. Yoshitake \\ A. Shinkawa, Y. Hasuo, M. Fujishima \\ The Second Department of Internal Medicine, Faculty of Medicine Kyushu University, Fukuoka, Japan
}

Summary To elucidate the risk factors for initiating glucose intolerance, the relevant factors were explored in a cross-sectional survey conducted in a sample population aged 40-79 years old selected from a Japanese community, Hisayama, Japan in 1988. A 75 -g oral glucose tolerance test was used to classify 1,073 men $(72.5 \%$ of the entire population in the same age range) and 1,407 women (80.5\%) into normal, impaired glucose tolerance and diabetes mellitus groups. In all age and sex groups with normal glucose tolerance, the sum of fasting and 2-h post-load insulin values varied widely and demonstrated significant positive correlations with triglycerides, body mass index, waist-hip ratio, systolic and diastolic blood pressure, while it negatively correlated to HDL cholesterol $(p<0.05)$. Insulin resistance was presumed to develop in normal glucose tolerance subjects with hyperinsulinaemia. The sum of the insulin concentrations, triglycerides, body mass index, waist-hip ratio and blood pressure levels was significantly associated with impaired glucose tolerance in all age and sex groups after adjustment for age $(p<0.05)$ and was also related to diabetes in either all or some age and sex groups, respectively $(p<0.05)$. It was shown that glucose intolerance in the general population was associated with the factors related to insulin resistance. These cross-sectional data, therefore, support the hypothesis that insulin resistance is the primary defect in the development of glucose intolerance in the Japanese general population. However, a further prospective study is still needed in order to confirm this hypothesis. [Diabetologia (1994) 37: 897-904]

Key words Insulin resistance, non-insulin-dependent diabetes mellitus, impaired glucose tolerance, population-based study, epidemiology, Japanese, Hisayama study.
There is controversy as to whether or not resistance to insulin-stimulated glucose uptake plays a primary role in the development of NIDDM $[1,2]$. Insulin resistance has been reported to be a risk factor in the initial development of glucose intolerance in Pima Indians, Nauruans and Mexican Americans, which are all well known as populations with a high prevalence

Received: 21 December 1993

and in revised form: 21 March 1994

Corresponding author: Dr. T. Ohmura, Second Department of Internal Medicine, Faculty of Medicine, Kyushu University, Maidashi 3-1-1. Higashi-ku, Fukuoka City 812, Japan

Abbreviations: OGTT, Oral glucose tolerance test; NIDDM, non-insulin-dependent diabetes mellitus. of NIDDM [3-7]. In this situation, beta-cell dysfunction is presumed to play a critical role in the later stages of deterioration of glucose intolerance $[5,8$, 9]. However, a reduction in the beta-cell mass has been demonstrated in the early stages of diabetes in the black population of southern Africa $[10,11]$. A study using continuous glucose infusion has suggested that beta-cell dysfunction is the primary cause for the initiation of familial NIDDM in Caucasians [12]. These conflicting findings indicate that there may be a difference in the pathogenesis of diabetes in ethnic groups.

This dispute has not been settled regarding the ethnic Japanese population. In Japanese-American men [13], the association of insulin resistance with the development of NIDDM has been demonstra- 
Table 1. The mean values and frequencies of the examined items by the level of glucose intolerance and sex for the subjects aged 40-59 years old; Hisayama, Japan, 1988

\begin{tabular}{|c|c|c|c|c|c|c|}
\hline & \multicolumn{3}{|l|}{ Men } & \multicolumn{3}{|l|}{ Women } \\
\hline & $\begin{array}{l}\text { Normal } \\
(n=451)\end{array}$ & $\begin{array}{l}\text { IGT } \\
(n=110)\end{array}$ & $\begin{array}{l}\text { Diabetes } \\
(n=86)\end{array}$ & $\begin{array}{l}\text { Normal } \\
(n=651)\end{array}$ & $\begin{array}{l}\text { IGT } \\
(n=136)\end{array}$ & $\begin{array}{l}\text { Diabetes } \\
(n=53)\end{array}$ \\
\hline Fasting insulin (pmol/l) & $30 \pm 10$ & $40^{\mathrm{a}} \pm 10$ & $45^{\mathrm{a}} \pm 10$ & $35 \pm 10$ & $40^{\mathrm{a}} \pm 10$ & $55^{a} \pm 10$ \\
\hline $2-\mathrm{h}$ post-load insulin (pmol/1) & $135 \pm 10$ & $325^{a} \pm 10$ & $220^{\mathrm{a}} \pm 15$ & $160 \pm 10$ & $285^{a} \pm 10$ & $290^{a} \pm 15$ \\
\hline Sum of insulin $(\mathrm{pmol} / \mathrm{l})$ & $170 \pm 10$ & $370^{\mathrm{a}} \pm 10$ & $275^{a} \pm 10$ & $200 \pm 10$ & $325^{\mathrm{a}} \pm 10$ & $355^{a} \pm 10$ \\
\hline Triglycerides $(\mathrm{mmol} / \mathrm{l})$ & $1.28 \pm 0.02$ & $1.76^{\mathrm{a}} \pm 0.02$ & $1.88^{\mathrm{a}} \pm 0.02$ & $0.94 \pm 0.02$ & $1.08^{\mathrm{a}} \pm 0.02$ & $1.42^{\mathrm{a}} \pm 0.02$ \\
\hline Body mass index $\left(\mathrm{kg} / \mathrm{m}^{2}\right)$ & $23.1 \pm 2.8$ & $24.4^{\mathrm{a}} \pm 3.0$ & $24.3^{\mathrm{a}} \pm 2.9$ & $23.0 \pm 3.0$ & $23.6 \pm 3.2$ & $25.3^{\mathrm{a}} \pm 4.2$ \\
\hline Waist-hip ratio & $0.92 \pm 0.05$ & $0.94^{\mathrm{a}} \pm 0.06$ & $0.95^{\mathrm{a}} \pm 0.04$ & $0.90 \pm 0.07$ & $0.92^{\mathrm{a}} \pm 0.07$ & $0.93^{\mathrm{a}} \pm 0.07$ \\
\hline Subscapular/triceps ratio & $2.2 \pm 1.4$ & $2.1 \pm 0.7$ & $2.1 \pm 0.6$ & $1.2 \pm 0.3$ & $1.2 \pm 0.3$ & $1.3^{\mathrm{a}} \pm 0.3$ \\
\hline Systolic BP (mm Hg) & $129 \pm 17$ & $138^{a} \pm 19$ & $141^{\mathrm{a}} \pm 18$ & $124 \pm 17$ & $131^{\mathrm{a}} \pm 18$ & $140^{\mathrm{a}} \pm 18$ \\
\hline Diastolic BP (mm Hg) & $81 \pm 11$ & $86^{a} \pm 12$ & $86^{\mathrm{a}} \pm 11$ & $76 \pm 11$ & $79^{\mathrm{a}} \pm 11$ & $82^{a} \pm 11$ \\
\hline
\end{tabular}

Values are means $\pm \mathrm{SD}$ or frequencies (\%) of each factor. ${ }^{\mathrm{a}} p<0.05,{ }^{\mathrm{b}} p<0.01$ (IGT or diabetes vs normal glucose tolerance). IGT, impaired glucose tolerance; BP, Blood pressure

ted, while a diminished insulin response was considered to be a risk factor for the deterioration to diabetes in clinical surveys of native Japanese out-patients who initially demonstrated mild glucose intolerance $[14,15]$. There have been no studies evaluating the contributing factors in the development of glucose intolerance from normal glucose tolerance in the native Japanese population.

We have already reported a much higher prevalence of NIDDM and impaired glucose tolerance in a Japanese community, Hisayama (the Hisayama study) than those previously reported in Japan [16]. In this study we investigate the important factors that contribute to the development of glucose intolerance from normal glucose tolerance, using cross-sectional data.

\section{Subjects and methods}

Subjects. The enrollment criteria, characteristics of the study population, and the overall design of the Hisayama study have been previously described in detail [16], wherein the population of the town of Hisayama was shown to be representative of the Japanese general population. We have studied the prevalence of diabetes based on data from a cross-sectional examination performed during 29 June -14 November 1988 . Of a total of 3,227 residents aged $40-79,2,587(80.2 \%)$ consented to participate.

The examination included an interview at which enquiries were made about diabetes and other previously diagnosed diseases and also about use of insulin and oral hypoglycaemic agents. All persons on insulin therapy had been diagnosed as having NIDDM by their attendant physicians. Personal drinking and smoking habits, as well as family history of diabetes were also assessed.

After the interviews, we excluded 92 participants from the study at the 75 -g OGTT, since 82 of them had eaten breakfast on the morning of examination and 10 were on insulin therapy for NIDDM. We could not complete the OGTT on 15 subjects because of complaints of nausea or general fatigue during the ingestion of glucose. Subjects receiving oral hypoglycaemic agents were requested to avoid taking their medication until completing the OGTT. Therefore, the OGTT was successfully completed on 1,073 men $(72.5 \%$ of the whole population in the same age range) and 1,407 women $(80.5 \%)$, in total.

Methods. Blood pressure was measured three times using a mercury sphygmomanometer (standard cuff size) with the subjects in the sitting position, and the average of the three values was used for the analyses.

Anthropometric measurements (height, weight, subscapular and triceps skinfold thickness, waist and hip circumferences) were made after the subjects had removed their clothes, shoes and socks. Using a Keys-Brosek skinfold caliper (Imoto Ikakikai Inc., Fukuoka, Japan), the subscapular skinfold thickness was measured just below the inferior angle of the right scapula. The triceps skinfold thickness was determined at the mid-line of the posterior aspect of the right arm, over triceps muscle, at the mid point between the acromial process of the scapula and the inferior margin of the olecranon process of the ulna. The waist circumference was measured at the level of the umbilicus. All measurements were taken after the subjects exhaled. The hip circumference was measured around the buttocks $4 \mathrm{~cm}$ below the anterior superior iliac spine. As a measure of obesity, the three following indices were used. The body mass index $\left(\mathrm{kg} / \mathrm{m}^{2}\right)$ was chosen as the indicator of whole-body obesity, the subscapular/triceps ratio for that of central adiposity, and waist-hip ratio for that of abdominal fat accumulation. Participants who either smoked cigarettes daily or drank alcohol daily were categorized as habitual smokers or drinkers. Diabetes in first or second degree relatives indicated a family history of diabetes.

Laboratory methods. The study subjects ingested glucose and other carbohydrate solutions equivalent to $75-\mathrm{g}$ of anhydrate glucose (Trelan G, Shimizu Pharmaceutical, Shimizu, Japan) between 08.00 and $10.30 \mathrm{a} . \mathrm{m}$. after at least a 12-h overnight 
Table 2. The mean values and frequencies of the examined items by the level of glucose intolerance and sex for the subjects aged 60-79 years old; Hisayama, Japan, 1988

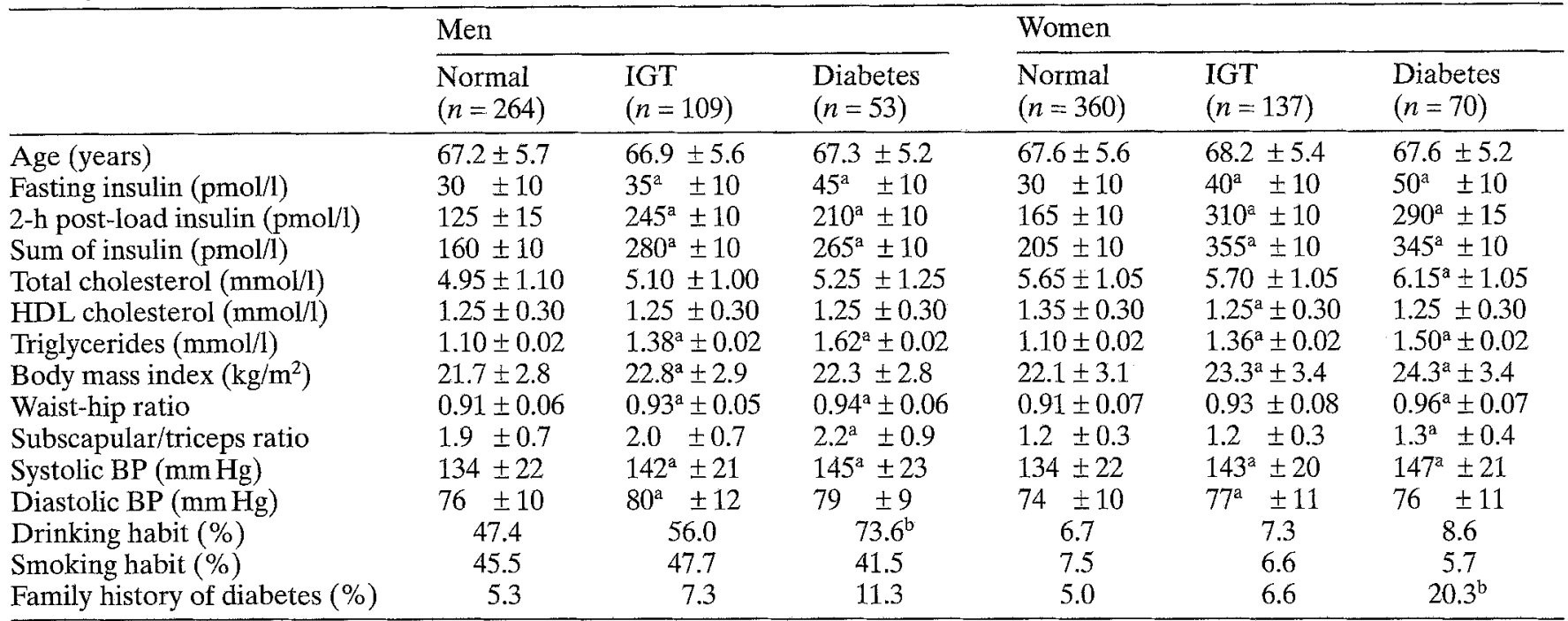

Values are means \pm SD or frequencies (\%) of each factor. ${ }^{\mathrm{a}} p<0.05,{ }^{\mathrm{b}} p<0.01$ (IGT or diabetes vs normal glucose tolerance).

IGT, Impaired glucose tolerance; BP, blood pressure

Table 3. Partial correlation analyses adjusting for age between insulin and other variables in the subjects with normal glucose tolerance

\begin{tabular}{|c|c|c|c|c|}
\hline \multirow[b]{3}{*}{ Sex } & \multicolumn{4}{|c|}{$\begin{array}{l}\text { Sum of insulin } \\
\text { Age (years) }\end{array}$} \\
\hline & \multicolumn{2}{|l|}{$40-59$} & \multicolumn{2}{|l|}{$60-79$} \\
\hline & $\begin{array}{l}\text { Men } \\
(n=451)\end{array}$ & $\begin{array}{l}\text { Women } \\
(n=651)\end{array}$ & $\begin{array}{l}\text { Men } \\
(n=264)\end{array}$ & $\begin{array}{l}\text { Women } \\
(n=360)\end{array}$ \\
\hline HDL cholestrol & $-0.19^{b}$ & $-0.29^{b}$ & $-0.16^{\mathrm{b}}$ & $-0.18^{b}$ \\
\hline Triglycerides & $0.26^{b}$ & $0.38^{b}$ & $0.26^{b}$ & $0.26^{b}$ \\
\hline Body mass index & $0.43^{\mathrm{b}}$ & $0.44^{\mathrm{b}}$ & $0.31^{\mathrm{b}}$ & $0.34^{\mathrm{b}}$ \\
\hline Waist-hip ratio & $0.34^{\mathrm{b}}$ & $0.36^{\mathrm{b}}$ & $0.13^{b}$ & $0.30^{\mathrm{b}}$ \\
\hline $\begin{array}{l}\text { Systolic blood } \\
\text { pressure }\end{array}$ & $0.13^{\mathrm{b}}$ & $0.21^{\mathrm{b}}$ & $0.21^{\mathrm{b}}$ & $0.16^{\mathrm{b}}$ \\
\hline $\begin{array}{l}\text { Diastolic blood } \\
\text { pressure }\end{array}$ & $0.21^{b}$ & $0.20^{b}$ & $0.18^{b}$ & $0.13^{\mathrm{a}}$ \\
\hline
\end{tabular}

${ }^{\mathrm{a}} p<0.05,{ }^{\mathrm{b}} p<0.01$

fast. To determine plasma glucose, serum insulin and lipid concentrations, blood was drawn twice from an antecubital vein before and 120 min after the ingestion of the glucose using simple vacutainer tubes and those with heparin, EDTA, and sodium fluoride. Blood specimens were transferred immediately after venupuncture to the central study laboratory (JML Inc., Fukuoka, Japan) in ice-cooled containers, where the tubes were centrifuged, and either the serum or plasma were separated. Plasma glucose was determined by the glucose-oxidase method using a Glucoroder-MK2 (A \& T Inc., Tokyo, Japan). The serum insulin level was determined by a commercial double-antibody solid-phase radioimmunoassay (Phadeseph Insulin; Pharmacia Diagnostics, Uppsala, Sweden). A fasting blood sample was used for the determination of lipid concentration. The total cholesterol, HDL cholesterol and triglycerides were all enzymatically determined using an autoanalyser (TBA-80S; Toshiba Inc., Tokyo, Japan). The determination of
HDL cholesterol was done after the precipitation of VLDL and LDL with dextran sulphate and magnesium.

According to World Health Organisation criteria [17], the subjects were divided into those with NIDDM, impaired glucose tolerance, and normal glucose tolerance. Inclusion into the diabetic group required fasting plasma glucose more than $7.8 \mathrm{mmol} / 1$ or a 2 -h plasma glucose value more than 11.1 $\mathrm{mmol} / \mathrm{l}$. The subjects with fasting plasma glucose less than $7.8 \mathrm{mmol} / \mathrm{l}$ and $\mathrm{a} 2$-h value between 7.8 and $11.1 \mathrm{mmol} / \mathrm{l}$ were categorized as having impaired glucose tolerance.

The average of fasting insulin values for the study subjects was low, and the frequency distribution curve was skewed toward the upper insulin level even after transformation into logarithms, while that of the integrated fasting and 2-h postload insulin levels showed a normal distribution curve (data not shown). Reaven et al. [18] and Hollenbeck et al. [19] reported a positive correlation between the integrated insulin levels during the OGTT and insulin resistance in normal glucose tolerance subjects. Referring to these studies, we utilized the sum of fasting and 2-h post-load insulin levels as an indicator for insulin resistance in subjects with normal glucose tolerance.

\section{Statistical analysis}

The SAS computer package (SAS Institute, Cary, N. C., USA) $[20,21]$ was used to perform all statistical analyses. Among the variables possibly related to glucose intolerance, age, insulin concentrations, systolic and diastolic blood pressure, lipid concentrations, body mass index, waist-hip ratio and subscapular/triceps ratio were all taken as continuous variables in the analyses. Drinking and smoking habits, and family history of diabetes were analysed as categorical data, and they were coded as either 1 or 0 according to the presence or absence of each factor, respectively. In order to correct skewed distribution, the insulin and triglyceride concentrations were transformed to logarithms, and then again were transformed to natural units after obtaining the means and standard deviations. The difference in the multiple comparisons of the mean values of continuous variables was evaluated by the Dunnett's $t$-test, 


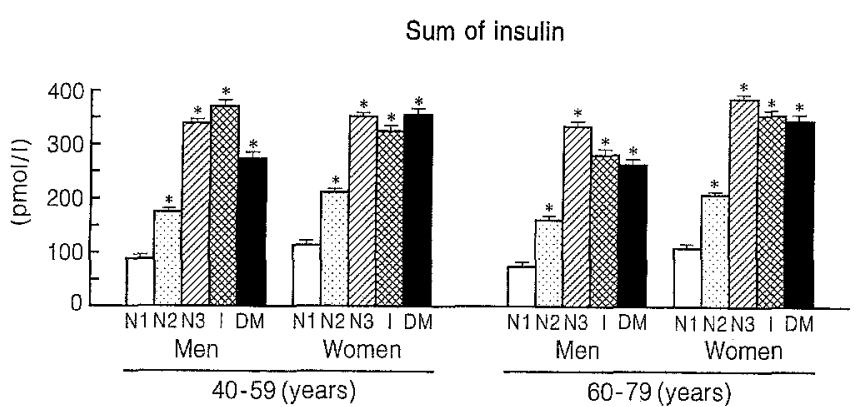

Fig. 1. The average values and standard deviation for serum insulin by either glucose intolerance or the insulin levels. The normal glucose tolerance subjects were divided into three groups according to the sum of fasting and 2-h post-load insulin levels. N1, the lowest; N2, the middle, N3, the highest tertile of the insulin levels in normal glucose tolerance. I, impaired glucose tolerance; DM, diabetes mellitus. * $\mathrm{p}<0.05$ (vs N1)
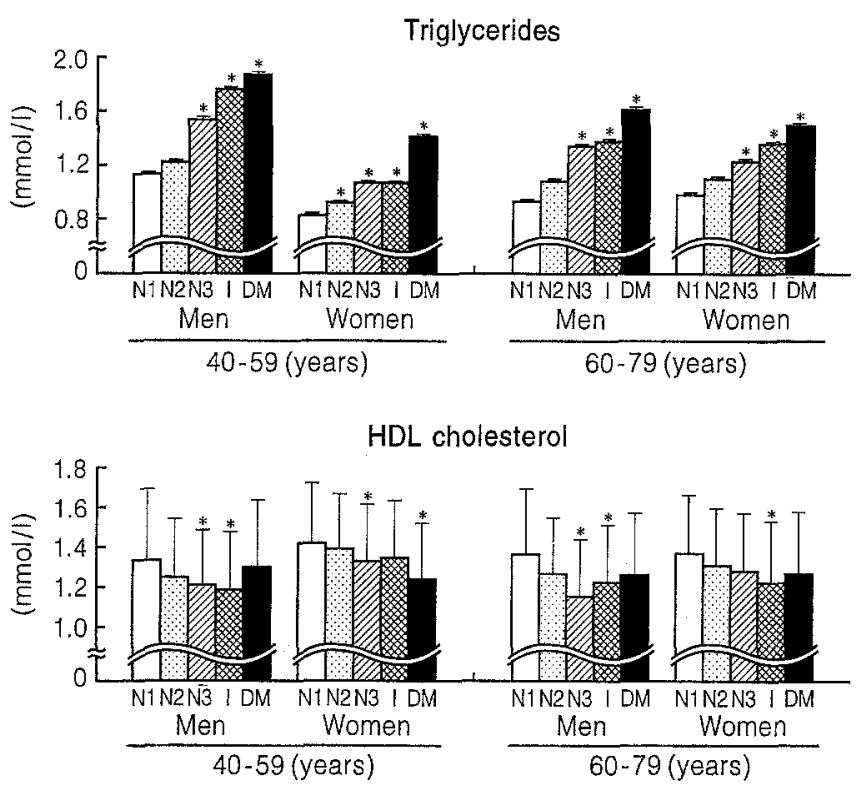

Fig. 2. The average values and standard deviation for serum lipids by either glucose intolerance or the insulin levels. The normal glucose tolerance subjects were divided into three groups according to the sum of fasting and $2-\mathrm{h}$ post-load insulin levels. N1, the lowest, N2, the middle, N3, the highest tertile of the insulin levels in normal glucose tolerance. I, impaired glucose tolerance. DM, diabetes mellitus. * $\mathrm{p}<0.05$ (vs N1)

and the difference in the frequency of categorical data was evaluated by the chi-square test (Table 1, Table 2, Figs. 1-4). To ascertain the relationship between the insulin values and the other variables, we used Spearman's rank correlation analyses taking age into account (Table 3 ).

Bivariate logistic regression analysis (age-adjustment) was utilized to evaluate how closely each variable was associated with either impaired glucose tolerance or diabetes, and the degree of the association was expressed by odds ratio (Table 4, Table 5). In analysing the factors related to impaired glucose tolerance, we assigned impaired glucose tolerance as code 1 for an objective variable in the logistic regression model and normal glucose tolerance as code 0 . While evaluating the factors associated with diabetes, the objective variable of diabetes was allocated to code 1 and that of normal glucose tolerance to code 0 .
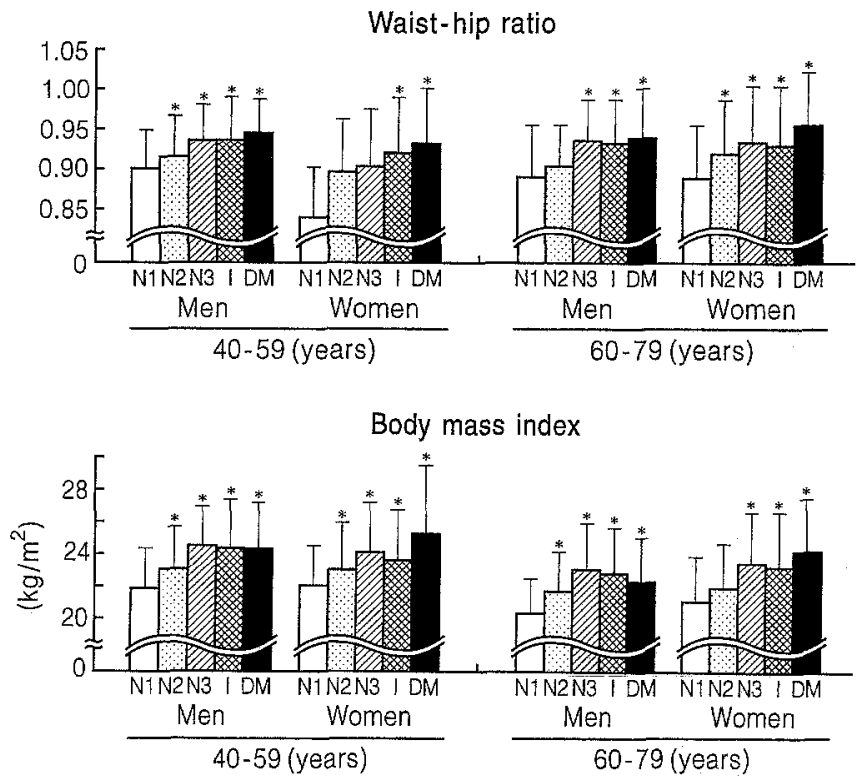

Fig. 3. The average values and standard deviation for the indices of obesity by glucose intolerance or the insulin levels. The normal glucose tolerance subjects were divided into three groups according to the sum of fasting and 2 -h post-load insulin levels. N1, the lowest, N2, the middle, N3, the highest tertile of the insulin levels in normal glucose tolerance. I, impaired glucose tolerance. DM, diabetes mellitus. $* p<0.05$ (vs N1)
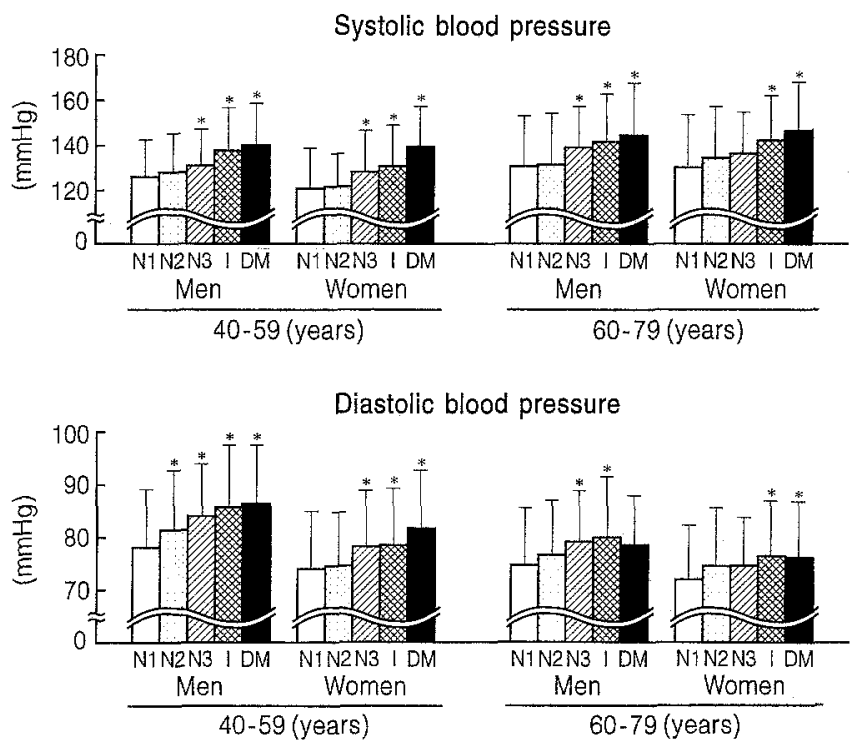

Fig. 4. The average values and standard deviation for blood pressure by glucose intolerance or the insulin levels. The normal glucose tolerance subjects were divided into three groups according to the sum of fasting and 2 -h post-load insulin levels. N1, the lowest, N2, the middle, N3, the highest tertile of the insulin levels in normal glucose tolerance. I, impaired glucose tolerance. DM, diabetes mellitus. * $\mathrm{p}<0.05$ (vs N1)

\section{Results}

We compared the mean values or frequencies of factors possibly related to glucose intolerance among the three groups, namely, the subjects with either im- 
Table 4. The age-adjusted odds ratio associated with impaired glucose tolerance for relevant factors by logistic regression analyses

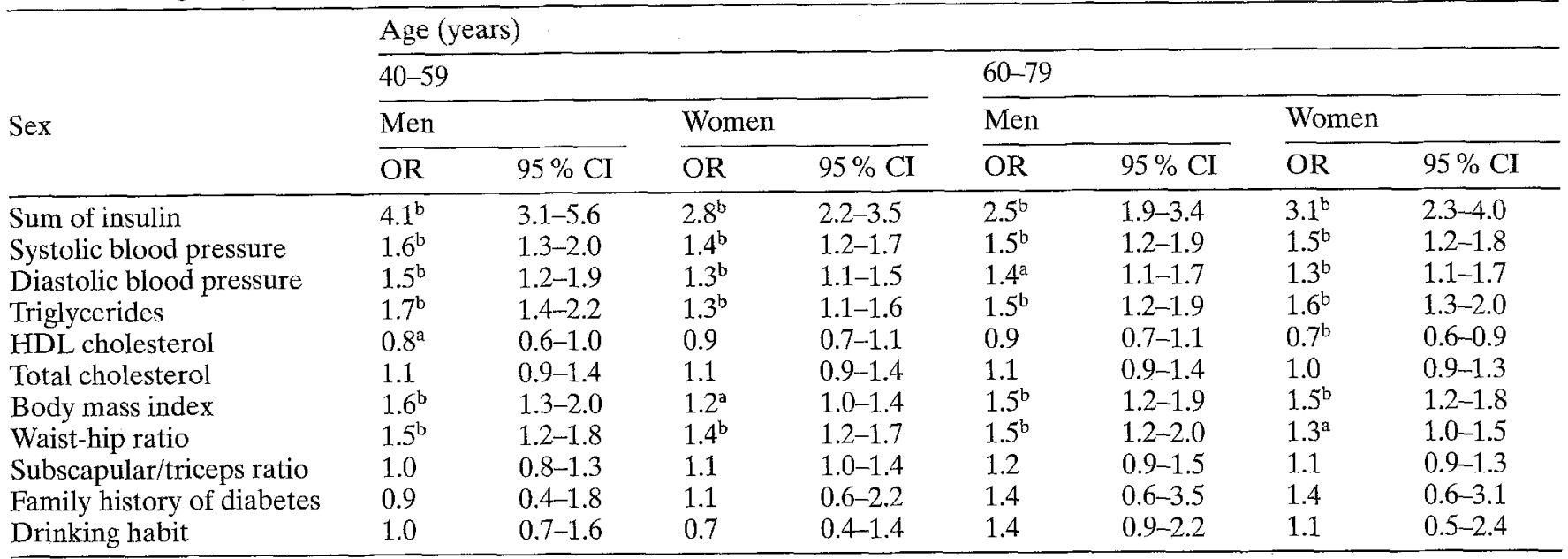

${ }^{\mathrm{a}} p<0.05,{ }^{\mathrm{b}} p<0.01$ for the association between impaired glucose tolerance and the related variable, controlling for age. Odds ratio (OR) of categorical factor is relative odds for subjects with impaired glucose tolerance compared with odds for those without glucose intolerance in a bivariate logistic regres- sion model with age as the only other independent variable. Odds ratio of a continuous variable is relative odds of impaired glucose tolerance associated with increase of the variable by one standard deviation in the bivariate model C. I., Confidence interval

Table 5. The age-adjusted odds ratio associated with diabetes for relevant factors by logistic regression analyses

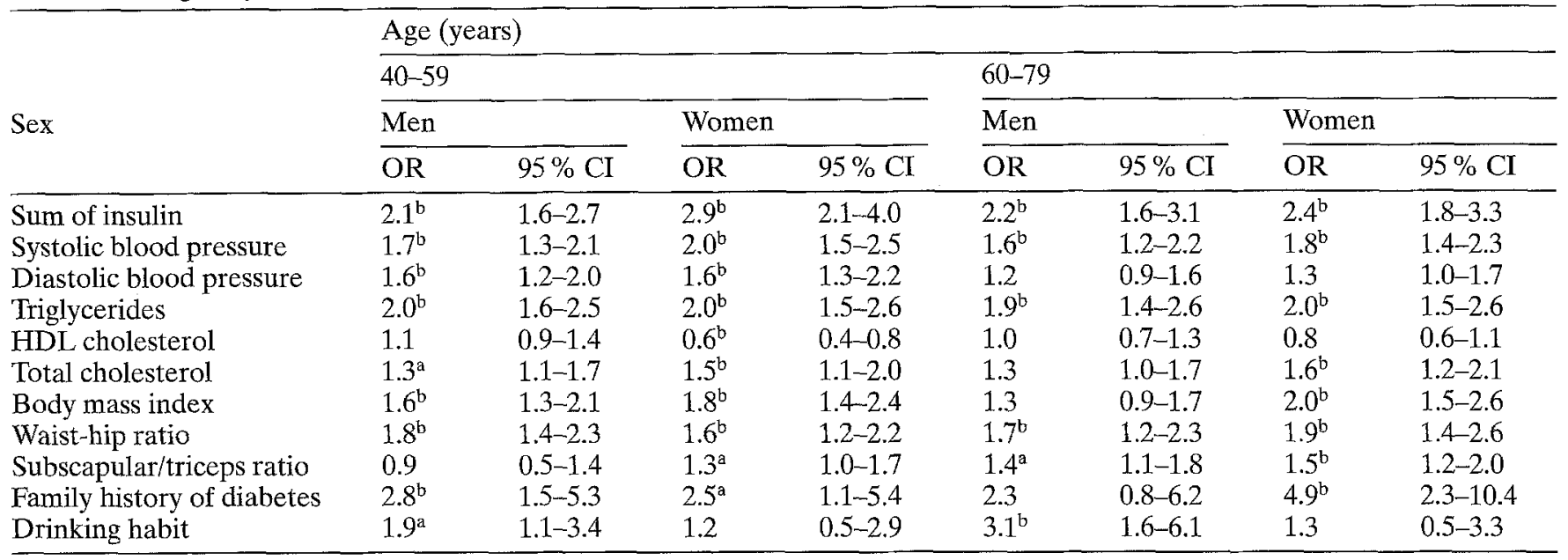

${ }^{\mathrm{a}} p<0.05,{ }^{\mathrm{b}} p<0.01$ for the association between diabetes and the related variable, controlling for age.

Odds ratio (OR) of categorical factor is relative odds for subjects with diabetes compared with odds for those without glucose intolerance in a bivariate logistic regression model with

paired glucose tolerance, diabetes or normal glucose tolerance by age and sex groups (Table 1, Table 2).

The fasting, 2-hour post-load insulin, triglyceride, systolic and diastolic blood pressure were all higher in subjects with impaired glucose tolerance than in those with normal glucose tolerance irrespective of age or $\operatorname{sex}(p<0.05)$. The waist-hip ratio was greater in subjects with impaired glucose tolerance aged 40 59 years, and the body mass index was larger in those aged $60-79$ years than those in the respective normal groups $(p<0.05)$.

Diabetic subjects demonstrated higher fasting and post-load insulin concentrations, higher trigly- age as the only other independent variable. Odds ratio of a continuous variable is relative odds of diabetes associated with increase of the variable by one standard deviation in the bivariate model

CI, Confidence interval ceride levels, and a greater waist-hip ratio and higher systolic blood pressure than normal subjects in all age and sex groups $(p<0.05)$. Subjects with diabetes also had a greater body mass index, a more elevated diastolic blood pressure, a higher frequency of family history of diabetes for those aged 4059 , and a greater subscapular/triceps ratio for those aged 60-79 than normal subjects in the respective age group $(p<0.05)$.

The age-adjusted partial correlation coefficients of the lipid levels, obesity and blood pressure with the sum of fasting and 2-h post-load insulin levels which reflects insulin resistance are shown for the subjects 
with normal glucose tolerance in Table 3. In all age and sex groups, triglyceride, body mass index, waisthip ratio, and systolic and diastolic blood pressure were all positively correlated with the sum of insulin $(p<0.05)$ and HDL cholesterol was negatively correlated $(p<0.05)$. These correlations suggest that the higher triglyceride levels, blood pressure, obesity and the lower HDL cholesterol levels were all related to insulin resistance.

In addition to impaired glucose tolerance and diabetes, we divided the normal glucose tolerance subjects into three groups on the basis of tertiles of the sum of fasting and 2-h post-load insulin values. The average levels of insulin, lipids, obesity and blood pressure were compared among these five groups (Figs. 1-4). The sum of fasting and 2-h post-load insulin levels varied more than threefold within the normal glucose tolerance groups. The insulin levels for the subjects classified into the highest tertile of the sum of insulin in normal glucose tolerance reached almost identical levels to those for either impaired glucose tolerance or diabetes in all age and sex groups (Fig. 1). The average values of triglycerides, waisthip ratio, body mass index, systolic and diastolic blood pressure for the group belonging to the highest tertile of insulin in the normal glucose tolerance group tended to be higher than those in the remaining subjects with normal glucose tolerance and close to those in the groups with either impaired glucose tolerance or diabetes irrespective of age and sex (Figs. 2-4). This evidence suggests that insulin resistance starts during the period of normal glucose tolerance, as the serum insulin level and the manifestations of insulin resistance such as raised triglyceride levels, obesity, and raised blood pressure in normal glucose tolerance subjects with high insulin values were close to those of glucose intolerance.

HDL cholesterol levels in diabetic men were higher than those in male subjects included into the highest tertile of insulin in normal glucose tolerance and similar to those for the remaining normal glucose tolerance subjects (Fig. 2).

To elucidate whether impaired glucose tolerance and diabetes are associated with the insulin resistance syndrome, we carried out bivariate logistic regression analyses on either impaired glucose tolerance or diabetes, where age and other factors related to glucose intolerance were subjected to the independent variables. Table 4 shows odds ratios related to impaired glucose tolerance for each variable after age-adjustment. The sum of fasting and 2-h postload insulin concentrations, systolic and diastolic blood pressure, triglycerides, body mass index and waist-hip ratio were all significantly related to impaired glucose tolerance in all sex and age groups $(p<0.05)$. Low HDL cholesterol levels had a significant effect on impaired glucose tolerance in men aged 40-59 years and women aged 60-79 years $(p<0.05)$. However, total cholesterol, subscapular/ triceps ratio, family history of diabetes, and drinking habits demonstrated no association with impaired glucose tolerance after age-adjustment for any sex or age group $(p>0.05)$.

We also examined the influence of various factors on diabetes by bivariate logistic regression analyses, while taking age into account. Table 5 shows the ageadjusted odds ratio for each variable related to diabetes. The sum of insulin, systolic blood pressure, triglycerides and the waist-hip ratio significantly increased the risk for diabetes in all age and sex groups $(p<0.05)$. Diastolic blood pressure, total cholesterol, body mass index, subscapular/triceps ratio, a family history of diabetes and drinking habits were also related to diabetes in some age or sex groups $(p<0.05)$. A low HDL cholesterol level only had a significant association with diabetes for women aged $40-59$ years $(p<0.05)$.

It was shown that insulin-resistant disorders including high triglyceride, an elevated blood pressure and obesity were commonly related to impaired glucose tolerance and to diabetes in both sexes.

\section{Discussion}

In order to examine whether or not insulin resistance is a risk factor for the initial development of glucose intolerance in the Japanese general population, we examined the association of the insulin resistance syndrome and glucose intolerance in a sample population of Hisayama residents.

The coexistence of glucose intolerance, dyslipidaemia, hypertension and obesity has been termed the 'deadly quartet' by Kaplan [22], and the clustering of glucose intolerance, hyperinsulinaemia, dyslipidaemia, hypertension on the basis of insulin resistance as 'syndrome X' by Reaven [23]. This insulinresistance syndrome was ascertained in a general population of Mexican Americans by the San Antonio Heart Study [24, 25].

Our data showed that, in subjects with normal glucose tolerance, hypertriglyceridaemia, obesity, higher blood pressure, and lower HDL cholesterol level were all significantly associated with higher insulin levels reflecting insulin resistance. Since these insulin resistant disorders were also related to both impaired glucose tolerance and diabetes in all or many age and sex groups in logistic regression analyses, glucose intolerance was considered to be associated with insulin resistance in the present study. The insulin levels in those with normal glucose tolerance varied widely and insulin resistance disorders tended to be accumulated in those with high insulin levels in the range of normal glucose tolerance. It seems rational to suggest that insulin resistance begins to increase in subjects with normal glucose tolerance who have 
high insulin values. These data thus support the hypothesis that insulin resistance is the primary defect in the development of NIDDM.

In logistic regression analyses, the insulin values were positively related to both impaired glucose tolerance and diabetes in all age and sex groups. In subjects with glucose intolerance, raised insulin levels after a glucose load might result not only from insulin resistance but also from either a delayed insulin response or elevated levels of proinsulin and other intermediates which cross-react with an insulin assay. However, since other factors reflecting insulin resistance, such as triglycerides, indices of obesity and blood pressure were also positively associated with impaired glucose tolerance and with diabetes, higher insulin levels might partially reflect the increased insulin resistance in subjects with glucose intolerance.

The HDL cholesterol levels in diabetic men were higher than those in the subjects belonging to the normal glucose tolerance group with high insulin levels. No association was observed in men between HDL cholesterol and diabetes in the logistic analyses of the present study. Since diabetic men tended to drink more, alcohol intake might elevate their HDL cholesterol in spite of their increased insulin resistance.

A family history of diabetes has been considered to be a risk factor for diabetes in many studies [26, 27-32], and the Paris Prospective Study [33] demonstrated that alcohol consumption was associated with the development of diabetes in men diagnosed by an OGTT. In our study, neither a family history of diabetes nor drinking habits were associated with impaired glucose tolerance in the logistic analyses, whereas these two factors were significantly related to diabetes in some age and sex groups. A family history of diabetes might be a manifestation of a gene expression which could worsen glucose intolerance, while alcohol ingestion could accelerate the progression from impaired glucose tolerance to diabetes.

Because of the cross-sectional design of this study, only an initial role for insulin resistance causing glucose intolerance could be shown. It is still unclear whether hyperinsulinaemia is a result or a predictor of glucose intolerance. Some prospective studies have shown multiple metabolic disorders such as hyperinsulinaemia or an elevated C-peptide level predicting the onset of glucose intolerance from non-diabetic subjects $[4-6,13,34]$. However, there have been no prospective studies in terms of the development of glucose intolerance in the Japanese general population with normal glucose tolerance. A further prospective study on the development of glucose intolerance in subjects with normal glucose tolerance would be needed particularly for the Japanese population, since the prevalence of diabetes is considered to be on the increase.
Acknowledgements. This study was supported in part by Grantin-Aid for Diabetes Epidemiology Research from The Ministry of Health and Welfare, Japan, and Funds for Research of Metabolic Disorders (Takeda), and was also facilitated by the Japan-U.S. Cooperative Agreement in the Cardiovascular Disease Center in Osaka, and the National Institutes of Health, Blood and Lung in Bethesda, Maryland, USA.

\section{References}

1. DeFronzo RA (1988) The triumvirate: beta cell, muscle, liver. A collusion responsible for NIDDM. Diabetes 37: 667-687

2. Hales CN, Barker DJP (1992) Type 2 (non-insulin-dependent) diabetes mellitus: the thrifty phenotype hypothesis. Diabetologia 35: 595-601

3. Haffner SM, Stern MP, Hazuda HP, Mitchell BD, Patterson JK (1988) Increased insulin concentrations in nondiabetic offspring of diabetic parents. N Engl J Med 319: 1297-1301

4. Knowler WC, Pettitt DJ, Saad MF, Bennett PH (1990) Diabetes mellitus in the Pima Indians: incidence, risk factors and pathogenesis. Diabetes Metab Rev 6: 1-27

5. Sicree RA, Zimmet PZ, King HOM, Coventry JS (1987) Plasma insulin response among Nauruans: prediction of deterioration in glucose tolerance over 6 years. Diabetes 36 : 179-186

6. Haffner SM, Stern MP, Mitchell BD, Hazuda HP, Patterson JK (1990) Incidence of type 2 diabetes in Mexican Americans predicted by fasting insulin and glucose levels, obesity, and body-fat distribution. Diabetes 39: 283-288

7. Boyko EJ, Keane EM, Marshall JA, Hamman RF (1991) Higher insulin and C-peptide concentrations in Hispanic population at high risk for NIDDM: San Luis Valley Diabetes Study. Diabetes 40: 509-515

8. Saad MF, Knowler WC, Pettit DJ, Nelson RG, Mott DM, Bennett PH (1988) The natural history of impaired glucose tolerance in the Pima Indians. N Engl J Med 319: $1500-1506$

9. Saad MF, Knowler WC, Pettit DJ, Nelson RG, Charles MA, Bennett PH (1991) A two-step model for development of non-insulin-dependent diabetes. Am J Med 90: 229-235

10. Wicks ACB, Jones JJ (1973) Insulinopenic diabetes in Africa. BMJ 31: 773-776

11. Joffe BI, Panz VR, Wing JR, Raal FJ, Seftel HC (1992) Pathogenesis of non-insulin-dependent diabetes mellitus in the black population of southern Africa. Lancet 340: 460462

12. O'Rahilly SP, Nugent Z, Rudenski AS et al. (1986) Betacell dysfunction, rather than insulin insensitivity, is the primary defect in familial type 2 diabetes. Lancet II: 360-364

13. Bergstrom RW, Newell-Morris LL, Leonetti DL, Shuman WP, Wahl PW, Fujimoto WY (1990) Association of elevated fasting C-peptide level and increased intra-abdominal fat distribution with development of NIDDM in JapaneseAmerican men. Diabetes 39: 104-111

14. Kosaka K, Hagura R, Kuzuya T (1977) Insulin responses in equivocal and definite diabetes, with special reference to subjects who had mild glucose intolerance but later developed definite diabetes. Diabetes 26: 944-952

15. Kadowaki T, Miyake Y, Hagura R et al. (1984) Risk factors for worsening to diabetes in subjects with impaired glucose tolerance. Diabetologia 26: 44-49

16. Ohmura T, Ueda K, Kiyohara Y et al. (1993) Prevalence of type 2 (non-insulin-dependent) diabetes mellitus and impaired glucose tolerance in the Japanese general population: the Hisayama study. Diabetologia 36: 1198-1203 
17. WHO study group on diabetes mellitus (1985) Diabetes mellitus, report of a WHO study group. World Health Organization (technical report series 727), Geneva

18. Reaven GM, Brand RJ, Chen YDI, Mathur AK, Goldfine I (1993) Insulin resistance and insulin secretion are determinants of oral glucose tolerance in normal individuals. Diabetes 42:1324-1332

19. Hollenbeck CB, Chen N, Chen YDI, Reaven GM (1984) Relationship between the plasma insulin response to oral glucose and insulin-stimulated glucose utilization in normal subjects. Diabetes 33: 460-463

20. SAS Institute (1990) SAS procedure guide, Version 6, third editon. SAS Insutitute, Cary NC

21. SAS Institute (1990) SAS/STAT user's gude, Version 6, fourth edition. SAS Institute, Cary NC

22. Kaplan NM (1989) The deadly quartet. Upper-body obesity, glucose intolerance, hypertriglyceridemia, and hypertension. Arch Intern Med 149: 1514-1520

23. Reaven GM (1988) Role of insulin resistance in human disease. Diabetes 37:1595-1607

24. Ferrannini E, Haffner SM, Mitchell BD Stern MP (1991) Hyperinsulinaemia: the key feature of a cardiovascular and metabolic syndrome. Diabetologia 34: 416-422

25. Haffner SM, Valdez RA, Hazuda HP, Mitchell BD, Morales PA, Stern MP (1992) Prospective analysis of the insulin-resistance syndrome (syndrome X). Diabetes 41: 715722

26. Marshall JA, Hamman RF, Baxter J et al. (1993) Ethnic differences in risk factors associated with the prevalence of non-insulin-dependent diabetes mellitus. The San Luis Valley Diabetes Study. Am J Epidemiol 137: 706-718
27. Knowler WC, Pettitt DJ, Savage PJ, Bennett PH (1981) Diabetes incidence in Pima Indians: contributions of obesity and parental diabetes. Am J Epidemiol 113: 144-156

28. Chou P, Chen HH, Hsiao KJ (1992) Community-based epidemiological study on diabetes in Pu-Li, Taiwan. Diabetes Care 15: $81-89$

29. Harris MI (1991) Epidemiological correlates of NIDDM in Hispanics, whites, and blacks in the U.S. population. Diabetes Care 14 (Suppl 3): 639-648

30. Mykkänen L, Laakso M, Uusitupa M, Pyörälä K (1990) Prevalence of diabetes and impaired glucose tolerance in elderly subjects and their association with obesity and family history of diabetes. Diabetes Care 13: 1099-1105

31. Ohlson LO, Larsson B, Björntorp P et al. (1988) Risk factors for type 2 (non-insulin-dependent) diabetes mellitus. Thirteen and one-half years of follow-up of the participants in a study of Swedish men born in 1913. Diabetologia 31: 798-805

32. Shaten BJ, Kuller LH, Smith GD, Neaton JD (1993) Risk factors for the development of type 2 diabetes among men enrolled in the usual care group of the multiple risk factor intervention trial. Diabetes Care 16: 1331-1339

33. Balkau B, Eschwège $E$, Fontbonne $A$, Claude JR, Warnet JM (1992) Cardiovascular and alcohol-related deaths in abnormal glucose tolerant and diabetic subjects. Diabetologia 35:39-44

34. Mykkänen L, Kuusisto J, Pyörälä K, Laakso M (1993) Cardiovascular disease risk factors as predictors of type 2 (non-insulin-dependent) diabetes mellitus in elderly subjects. Diabetologia 36: 553-559 